\title{
A New Family of Distributions Based on the Generalized Pearson Differential Equation with Some Applications
}

\author{
Mohammad Shakil ${ }^{1}$, B. M. Golam Kibria ${ }^{2}$ and Jai Narain Singh $^{3}$ \\ ${ }^{1}$ Department of Mathematics, Miami Dade College, USA \\ ${ }^{2}$ Department of Mathematics and Statistics, Florida International, USA \\ ${ }^{3}$ Department of Mathematics and Computer Science, Barry, USA
}

\begin{abstract}
Recently, a generalization of the Pearson differential equation has appeared in the literature, from which a vast majority of continuous probability density functions (pdf's) can be generated, known as the generalized Pearson system of continuous probability distributions. This paper derives a new family of distributions based on the generalized Pearson differential equation, which is a natural generalization of the generalized inverse Gaussian distribution. Some characteristics of the new distribution are obtained. Plots for the cumulative distribution function, pdf and hazard function, tables with percentiles and with values of skewness and kurtosis are provided. It is observed that the new distribution is skewed to the right and bears most of the properties of skewed distributions. As a motivation, the statistical applications of the results to a problem of forestry have been provided. It is found that our newly proposed model fits better than gamma, log-normal and inverse Gaussian distributions. Since many researchers have studied the use of the generalized inverse Gaussian distributions in the fields of biomedicine, demography, environmental and ecological sciences, finance, lifetime data, reliability theory, traffic data, etc., we hope the findings of the paper will be useful for the practitioners in various fields of theoretical and applied sciences.
\end{abstract}

Zusammenfassung: Neuerdings ist in der Literatur eine Verallgemeinerung der Pearson Differentialgleichung erschienen, von der eine große Anzahl stetiger Dichtefunktionen abgeleitet werden kann, die als generalisiertes Pearson System stetiger Verteilungsfunktionen bekannt sind. In diesem Artikel wird eine neue Familie von Verteilungen hergeleitet, welche auf der generalisierten Pearson Differentialgleichung beruht und die eine natürliche Verallgemeinerung der generalisierten inversen Gauss-Verteilung ist. Einige Eigenschaften dieser neuen Verteilung werden hergeleitet. Plots der Verteilungsfunktion, Dichtefunktion und der Hazardfunktion, Tabellen mit den Perzentilen und mit Werten der Schiefe und der Kurtosis werden angeboten. Es fällt auf, dass die neue Verteilung rechtsschief ist und die meisten Eigenschaften von schiefen Verteilungen aufweist. Als Motivation werden die Resultate auf ein Problem in der Forstwirtschaft angewandt. Dabei passt unser neues Modell besser als eine Gamma-, log-normal und inverse GaussVerteilung. Da viele Forscher den Gebrauch von generalisierten inversen Gauss-Verteilungen in der Biomedizin, Demographie, Umweltwissenschaft, Finanzwesen, bei Lebensdauer- und Verkehrsuntersuchungen, etc., untersucht 
haben, hoffen wir, dass unsere Ergebnisse für Praktiker in den verschiedenen Fächern der theoretischen und angewandten Wissenschaften von Nutzen sein werden.

Keywords: Generalized Inverse Gaussian Distribution, Goodness-of-Fit, Modified Bessel Function, Pearson Generalized Differential Equation.

\section{Introduction}

Recently, a generalization of the Pearson differential equation has appeared in the literature:

$$
\frac{d f_{X}(x)}{d x}=\frac{a_{0}+a_{1} x+a_{2} x^{2}+\cdots+a_{m} x^{m}}{b_{0}+b_{1} x+b_{2} x^{2}+\cdots+b_{n} x^{n}} f_{X}(x),
$$

where $m, n \geq 1$ are arbitrary integers, and the coefficients $a$ and $b$ are real numbers. By proper choice of the parameters $a$ and $b$, a vast majority of continuous probability density functions (pdf's) can be generated from equation (1), known as the generalized Pearson system of continuous probability distributions. Note that the classical differential equation introduced by Karl Pearson during the late 19th century is a special case of (1). For details on the Pearson system of continuous probability distributions, the interested readers are referred to Elderton (1953), Stuart and Ord (1994), and Johnson, Kotz, and Balakrishnan (1994), among others. The well-known families of continuous probability distributions such as the normal and the Student $t$ distributions (known as Pearson Type VII), beta distribution (known as Pearson Type I), and gamma distribution (known as Pearson Type III), introduced by Karl Pearson during the late 19th century can be generated as a solution to (1) by a proper choice of the parameters. For example, the normal distribution belongs to the generalized Pearson system of continuous probability distributions when $m=1$, $n=2, a_{0}=-\mu, a_{1}=1, b_{0}=-\sigma^{2}, b_{1}=0, b_{2}=0$. It appears from the literature that not much attention has been paid to the study of the family of continuous pdf's that can be generated as a solution to the generalized Pearson differential equation (1), except Dunning and Hanson (1977), Chaudhry and Ahmad (1993), and recently Shakil, Singh, and Kibria (2010). In Dunning and Hanson (1977), a generalization of the Pearson curves has been obtained as solution of (1) which best fits a histogram in the mean square sense and satisfies certain statistical constraints. Chaudhry and Ahmad (1993) have introduced a distribution with the following pdf as a solution to equation (1)

$$
f_{X}(x)=2 \sqrt{\frac{\alpha}{\pi}} \exp \left[-\left\{\sqrt{\alpha} x-\sqrt{\beta} x^{-1}\right\}^{2}\right], \quad \alpha>0, \beta>0, x>0,
$$

when $m=4, n=3, b_{0}=b_{1}=b_{2}=0, a_{4} / 2 b_{3}=-2 \alpha, a_{0} / 2 b_{3}=2 \beta, b_{3} \neq 0$, which defines the root reciprocal inverse Gaussian distribution, that is, the distribution of the random variable $X=1 / \sqrt{Y}$, where $Y$ has an inverse Gaussian (IG) distribution. The mode of Chaudhry and Ahmad's pdf (2) is at $x=(\beta / \alpha)^{1 / 4}$, and is skewed to the right. This paper derives a new family of continuous pdf's as a solution to (1), which includes the $p$-th root reciprocal of the IG distribution, that is, the distribution of the random variable $X=1 / \sqrt[p]{Y}$, where $Y$ has an IG distribution. It will be seen that this new distribution 
is more flexible and is a natural generalization of the IG and the generalized inverse Gaussian (GIG) distributions. It has also been observed that a number of other distributions including those of Chaudhry and Ahmad (1993) and Chou and Huang (2004) are special cases of this distribution. For some discussions on IG and GIG, the interested readers are referred to Jørgensen (1982), Johnson et al. (1994), and Chou and Huang (2004), among others. In what follows, some characteristics of our newly proposed distribution, including the expressions for the normalizing constant, pdf, cumulative distribution function (cdf), $k$-th moment, Shannon's entropy and relationships to other probability distributions, are derived. The plots for the cdf and pdf of the new distribution, including the percentile points, for some selected values of parameters, have been provided. The infinite divisibility property of the newly proposed distribution family is discussed. The distributional relationships to some distributions are established. The plots for the cdf, pdf and hazard function, percentile points and tables for Pearson's measures of skewness and kurtosis for selected coefficients and parameters have been provided. The estimation of parameters by maximum likelihood estimation and method of moments are discussed. It is observed that the new distribution is skewed to the right and bears most of the properties of skewed distributions. Since many researchers have studied the uses of the IG and the GIG distributions in the fields of biomedicine, demography, environmental and ecological sciences, lifetime data, reliability theory, traffic data, etc., we hope the findings of the paper will be useful for the practitioners in various fields of theoretical and applied sciences.

The organization of this paper is as follows. In Section 2, the pdf and cdf of the proposed distribution have been provided. Section 3 discusses some characteristics of the new distribution. Some distributional relationships are presented in Section 4. The percentage points of the new distribution are given in Section 5. The statistical applications of the results are contained in Section 6. Some concluding remarks are provided in Section 7. The derivations of the cdf, pdf, $k$-th moment, etc, in this paper involve some special functions, which are provided in the Appendix.

\section{Derivation of the New Probability Distribution}

In this section, the new continuous pdf is derived as a solution to the generalized Pearson differential equation (1). The expression for the cdf of the new distribution is obtained. Some graphical representations of the pdf and cdf of the new distribution for some selected values of the parameters are provided.

\subsection{Expressions for the Normalizing Constant and for the PDF}

We consider the generalized Pearson differential equation (1) in the following form

$$
\frac{d f_{X}(x)}{d x}=\frac{a_{0}+a_{p} x^{p}+a_{2 p} x^{2 p}}{b_{p+1} x^{p+1}} f_{X}(x), \quad b_{p+1} \neq 0, x>0,
$$

when $m=2 p, n=p+1, a_{1}=a_{2}=\cdots=a_{p-1}=a_{p+1}=\cdots=a_{2 p-1}=0$ and $b_{0}=b_{1}=b_{2}=\cdots=b_{p}=0$. The solution to the differential equation (3) is given by

$$
f_{X}(x)=C x^{\nu-1} \exp \left(-\alpha x^{p}-\beta x^{-p}\right), \quad x>0, \alpha \geq 0, \beta \geq 0, \nu \in \mathbb{R},
$$


where $\alpha=-\left(a_{2 p}\right) / p b_{p+1}, \beta=a_{0} / p b_{p+1}, \nu=\left(a_{p}+b_{p+1}\right) / b_{p+1}, b_{p+1} \neq 0, p>0$, and $C$ is the normalizing constant. According to the parameters $\{\alpha, \beta, \nu, p\}$, our newly proposed family of generalized GIG (GGIG) distributions may be classified into the following three classes, for which, using Lemma 1 and definition of gamma function (see the Appendix), the respective normalizing constants are also easily evaluated as given below:

1. Class I: $\alpha>0, \beta>0, \nu \in \mathbb{R}$, and $p>0$;

$$
C=\frac{p}{2}\left(\frac{\alpha}{\beta}\right)^{\nu / 2 p} \frac{1}{K_{\nu / p}(2 \sqrt{\alpha \beta})},
$$

where $K_{\nu / p}(2 \sqrt{\alpha \beta})$ denotes the modified Bessel function of third kind (see the Appendix).

2. Class II: $\alpha>0, \beta=0, \nu>0$, and $p>0$;

$$
C=\frac{p(\alpha)^{\nu / p}}{\Gamma(\nu / p)}
$$

3. Class III: $\alpha=0, \beta>0, \nu<0$, and $p>0$;

$$
C=\frac{p}{\beta^{\nu / p} \Gamma(-\nu / p)} .
$$

\section{Special Cases of the GGIG Distribution:}

It is easy to see that, by a simple transformation of the variable $x$ or by taking special values of the parameters $\{\alpha, \beta, \nu, p\}$, a number of distributions such as the IG (with $p=1$, $\nu=-1 / 2$ ), GIG (with $p=1$ ), reciprocal IG (with $p=1, \nu=1 / 2$ ), hyperbolic (with $p=1, \nu=1$ ), hyperbola (with $p=1, \nu=0$ ), generalized gamma (with $\alpha=(1 / \mu)^{p}$, $\beta=0, \nu=p k$, where $\mu>0, k>0$, see Achcar and Bolfarine, 1986), and pdf for other well-known distributions such as chi-squared (with $\alpha=1 / 2, \beta=0, p=1, \nu=k / 2$, where $k>0$ is an integer), chi (with $\alpha=1 / 2, \beta=0, p=2, \nu=k$, where $k>0$ is an integer), Erlang (with $\beta=0, p=1, \nu=c$, where $c>0$ is an integer), exponential (with $p=1, \nu=1, \beta=0$ ), gamma (with $p=1, \beta=0$ ), Weibull (with $p=\nu, \beta=0$, $\alpha=(1 / \eta)^{\nu}$, where $\eta>0$ ), Rayleigh (with $p=2, \nu=2, \beta=0, \alpha=1 / 2 \eta^{2}$ ), MaxwellBoltzman (with $p=2, \nu=3, \beta=0, \alpha=1 / 2 \eta^{2}$ ), half-normal (with $p=2, \nu=1$, $\left.\beta=0, \alpha=1 / 2 \eta^{2}\right)$, log-normal (with $\alpha=(1 / \mu)^{p}, \beta=0, \nu=p k$, where $\mu>0, k>0$ ), and letting $k \rightarrow \infty$ (see Lawless, 2003), inverse gamma (with $p=1, \alpha=0, \nu=-\tau$, where $\tau>0$ ), inverse half-normal (with $p=2, \alpha=0, \nu=-1, \beta=\theta^{2} / \pi$, where $\theta>0$ ), among others, can be derived as special cases from the equation (4), belonging to one of the classes as defined above.

It is also worth noting that our family of GGIG distributions is closed under the power transformation. That is, if $X \sim \operatorname{GGIG}(\alpha, \beta, \nu, p)$, then $Y=X^{s} \sim \operatorname{GGIG}(\alpha, \beta, \nu / s, p / s)$, where $s>0$. One can use this property in information analysis, for which the interested readers are referred to Dadpay, Soofi, and Soyer (2007), where similar properties have been considered in the context of the generalized gamma distribution family. This paper considers Class I as it is more general than the other two classes. Thus, from (4) and (5), 
for a random variable $X$, the following continuous pdf in terms of the modified Bessel function of the third kind is generated from the generalized Pearson differential equation (3):

$$
f_{X}(x)=\frac{p}{2}\left(\frac{\alpha}{\beta}\right)^{\frac{\nu}{2 p}} \frac{x^{\nu-1} \exp \left(-\alpha x^{p}-\beta x^{-p}\right)}{K_{\nu / p}(2 \sqrt{\alpha \beta})},
$$

where $x>0, \alpha>0, \beta>0, \nu \in \mathbb{R}$, and $p>0$. We refer to this as the GGIG distribution family. Note that $\nu$ and $p$ are shape parameters, and $\alpha$ and $\beta$ denote scale parameters. Using the definition of the Whittaker function (see appendix A), equation (6) is easily expressed as

$$
f_{X}(x)=\frac{p}{\sqrt{\pi}}\left(\frac{\alpha^{2 \nu+p}}{\beta^{2 \nu-p}}\right)^{\frac{1}{4 p}} \frac{x^{\nu-1} \exp \left(-\alpha x^{p}-\beta x^{-p}\right)}{W_{0, \nu / p}(4 \sqrt{\alpha \beta})}, \quad \alpha>0, \beta>0, x>0, p>0 .
$$

Remark 1: In view of the facts that GIG is used in the area of finance (as mixing distribution) in the context of the generalized hyperbolic distribution family (see e.g. Prause, 1997, 1999), and as our proposed distribution family is a generalization of the GIG distribution (which we refer to as the GGIG distribution family), it is hoped that one can use the GGIG in the area of finance and other fields of statistical research.

Remark 2: (Infinite Divisibility of the GGIG Distribution Family) Note that when $p=1$ and $\nu \in \mathbb{R}$, equation (6) reduces to the pdf of the GGIG distribution. The infinite divisibility of the GIG distribution has been determined by Barndorff-Nielson and Halgreen (1977), for which the interested readers are also referred to Marshall and Olkin (2007, p. 466) or Theorem 5.22 of Steutel and Harn (2004, p. 361). On the other hand, if $p>1$, then by Theorem 9.1 of Steutel and Harn $\left(2004\right.$, p. 115) it follows that $f_{X}(x)$ is not infinitely divisible.

\subsection{Derivation of the CDF}

Suppose $X$ is a random variable with the pdf $f_{X}(x)$ as given in (6). Then, using the definitions of exponential and incomplete gamma functions, the cdf of the random variable $X$ can easily be expressed as

$$
\begin{aligned}
F_{X}(x) & =\operatorname{Pr}(X \leq x)=\int_{0}^{x} f_{X}(x) d x \\
& =\frac{1}{2(\alpha \beta)^{\nu / 2 p} K_{\nu / p}(2 \sqrt{\alpha \beta})} \sum_{k=0}^{\infty} \frac{(-1)^{k}(\alpha \beta)^{k}}{k !} \gamma\left(\frac{\nu-p k}{p}, \alpha x^{p}\right),
\end{aligned}
$$

where $\alpha>0, \beta>0, \nu \in \mathbb{R}, p>0$. By direct differentiation of the cdf in (7) and noting that $\partial \gamma(a, t) / \partial t=t^{a-1} e^{-t}$, it can be easily verified that $d F_{X}(x) / d x=f_{X}(x)$, where $f_{X}(x)$ denotes the pdf of the random variable $X$ as given in (6). Using the series expansion of $\exp \left(-b t^{-1}\right)$ in the definition of the generalized incomplete gamma function, the following result easily follows:

$$
\gamma(\eta, z ; b)=\int_{0}^{z} t^{\eta-1} \exp \left(-t-b t^{-1}\right) d t=\sum_{k=0}^{\infty} \frac{(-b)^{k}}{k !} \gamma(\eta-k, z)
$$


where $\gamma(\eta-k, z)$ denotes the ordinary incomplete gamma function. Thus, using (8) in equation (7), the following expression for the cdf is obtained:

$$
F_{X}(x)=\frac{\gamma\left(\frac{\nu}{p}, \alpha x^{p} ; \alpha \beta\right)}{2(\alpha \beta)^{\nu / 2 p} K_{\nu / p}(2 \sqrt{\alpha \beta})}, \quad \alpha>0, \beta>0, \nu \in \mathbb{R}, p>0 .
$$

Using the definition of Whittaker function, the cdf (9) is easily expressed as

$$
F_{X}(x)=\frac{\gamma\left(\frac{\nu}{p}, \alpha x^{p} ; \alpha \beta\right)}{\sqrt{\pi}(\alpha \beta)^{\frac{2 \nu-p}{4 p}} W_{0, \nu / p}(4 \sqrt{\alpha \beta})}, \quad \alpha>0, \beta>0, \nu \in \mathbb{R}, p>0 .
$$

As a special case of equation (6) for $\nu=1, p=2$, by substituting $t=\sqrt{z / \alpha}$ in the following integral, and applying the Lemma 2 (see the Appendix), the cdf of the random variable $X$ is easily expressed in terms of the generalized incomplete gamma and error functions as follows:

$$
\begin{aligned}
F_{X}(x) & =2 \sqrt{\frac{\alpha}{\pi}} \exp (2 \sqrt{\alpha \beta}) \int_{0}^{x} \exp \left(-\alpha t^{2}-\beta t^{-2}\right) d t \\
& =\frac{1}{\sqrt{\pi}} \exp (2 \sqrt{\alpha \beta}) \gamma\left(\frac{1}{2}, \alpha x^{2} ; \alpha \beta\right) \\
& =1-\frac{1}{\sqrt{\pi}} \exp (2 \sqrt{\alpha \beta}) \Gamma\left(\frac{1}{2}, \alpha x^{2} ; \alpha \beta\right) \\
& =1-\frac{1}{2}\left[\operatorname{erfc}\left(\sqrt{\alpha} x-\sqrt{\beta} x^{-1}\right)+\exp (4 \sqrt{\alpha \beta}) \operatorname{erfc}\left(\sqrt{\alpha} x+\sqrt{\beta} x^{-1}\right)\right]
\end{aligned}
$$

where $\alpha>0, \beta>0$. Further, applying the equations (2.130) and (2.131) of Chaudhry and Zubair (2002, p. 53), the cdf of $X$, given in (12), can easily be expressed in terms of the incomplete gamma and confluent hypergeometric functions respectively as follows:

$$
F_{X}(x)=1-\frac{1}{2 \sqrt{\pi}}\left[\Gamma\left(\frac{1}{2}, u\right)+\exp (4 \sqrt{\alpha \beta}) \Gamma\left(\frac{1}{2}, v\right)\right],
$$

and

$$
F_{X}(x)=1-\frac{1}{\sqrt{\pi}} \exp (2 \sqrt{\alpha} \beta) \exp \left(-\alpha x^{2}-\beta x^{-2}\right)\left[\psi\left(\frac{1}{2}, \frac{1}{2} ; u\right)+\psi\left(\frac{1}{2}, \frac{1}{2} ; v\right)\right],
$$

where $u=\alpha x^{2}+\beta x^{-2}-2 \sqrt{\alpha \beta}$, and $v=\alpha x^{2}+\beta x^{-2}+2 \sqrt{\alpha \beta}, x>0, \alpha>0, \beta>0$.

Noting that $\operatorname{erfc}(z / \sqrt{2})=1-2 \Phi(z)$, where $\Phi(z)$ denotes the cdf of the standard normal distribution, the cdf of the random variable $X$, given by the equations (13), is easily be expressed in terms of $\Phi$ as

$$
\begin{aligned}
F_{X}(x)= & \frac{1}{2}[1-\exp (4 \sqrt{\alpha \beta})]+\Phi\left(\sqrt{2}\left(\sqrt{\alpha} x-\sqrt{\beta} x^{-1}\right)\right) \\
& +\exp (4 \sqrt{\alpha \beta}) \Phi\left(\sqrt{2}\left(\sqrt{\alpha} x+\sqrt{\beta} x^{-1}\right)\right),
\end{aligned}
$$

where $x>0, \alpha>0, \beta>0$. Applying theorem 2.8 of Chaudhry and Zubair (2002, p. 57) and equation 28 of Erdélyi, Magnus, Oberhettinger, and Tricomi (1953, p. 226), the cdf of $X$, given by the equations (11) or (12), is expressed as

$$
F_{X}(x)=1-\frac{1}{\sqrt{\pi}} \exp (2 \sqrt{\alpha \beta})\left[\Gamma\left(\frac{1}{2}\right)_{0} F_{1}\left(\frac{1}{2} ; \alpha \beta\right)-2 \sqrt{\alpha} x \Gamma_{2}\left(-\frac{1}{2}, \frac{1}{2}, \alpha x^{2}, \beta x^{-2}\right)\right],
$$

where $\Gamma_{2}(\cdot)$ denotes Horn's hypergeometric series of two variables. 


\subsection{Plots of the PDF and CDF of the Random Variable $X$}

The possible shapes of the pdf (6) and cdf (9) of $X$ are provided for some selected values of the parameters in Figures 1 to 4, respectively. The effects of the parameters can easily be seen from these graphs. For example, it is clear from the plotted Figures 1 to 2, for selected values of the parameters, the distributions of $X$ are positively (that is, right) skewed with longer and heavier right tails.
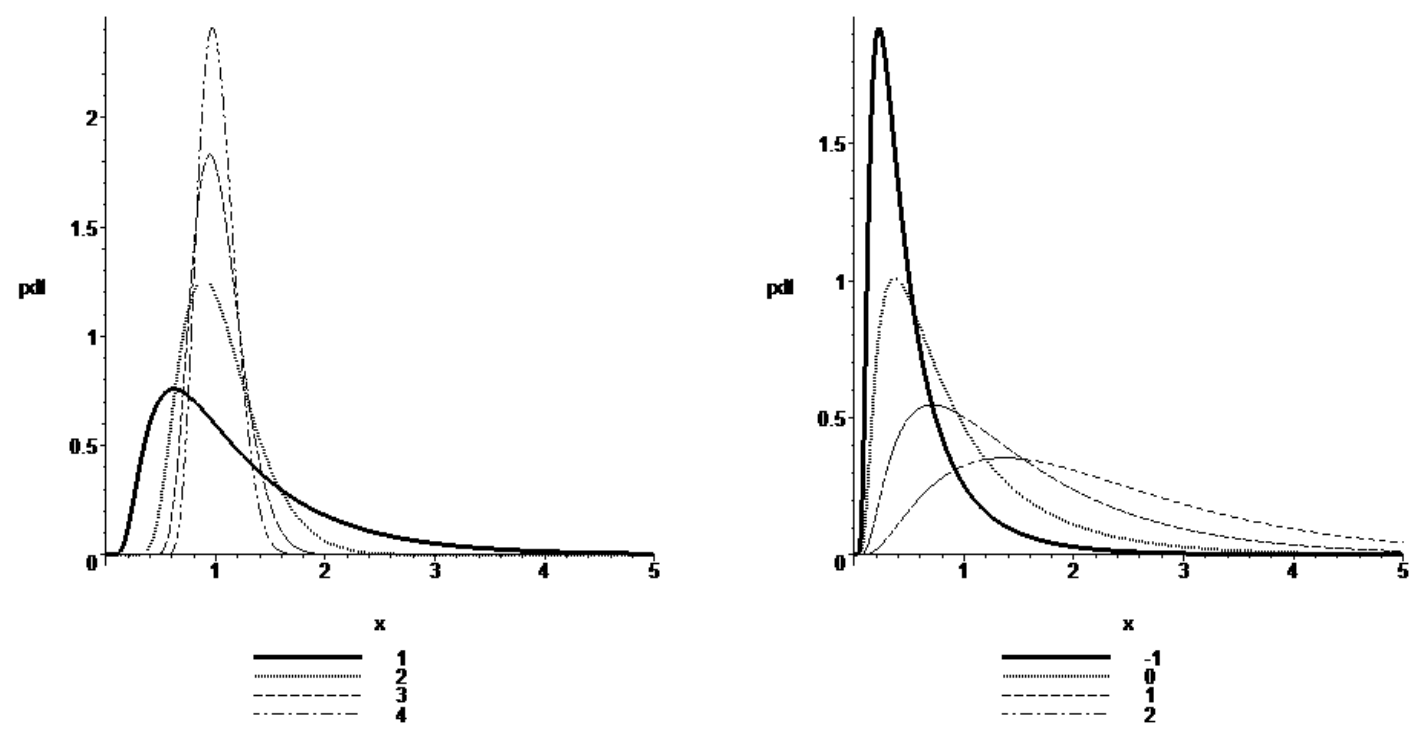

Figure 1: PDF plots of $X$ for $\alpha=1, \beta=1, \nu=0, p=1,2,3,4$ (left), and for $\alpha=1$, $\beta=1 / 2, p=1, \nu=-1,0,1,2$ (right).
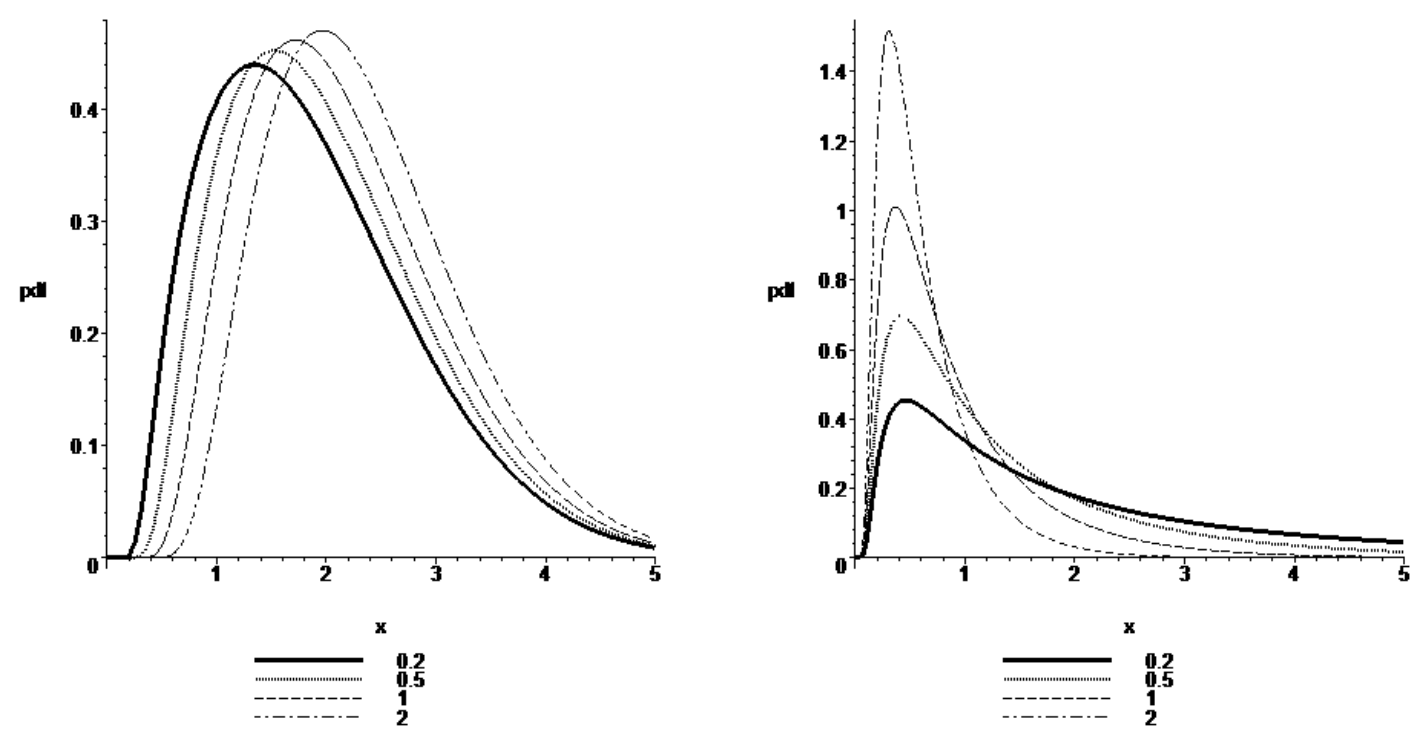

Figure 2: PDF plots of $X$ for $\alpha=0.2, \nu=3 / 2, p=2, \beta=0.2,0.5,1,2$ (left), and for $\beta=1 / 2, \nu=0, p=1, \alpha=0.2,0.5,1,2$ (right). 

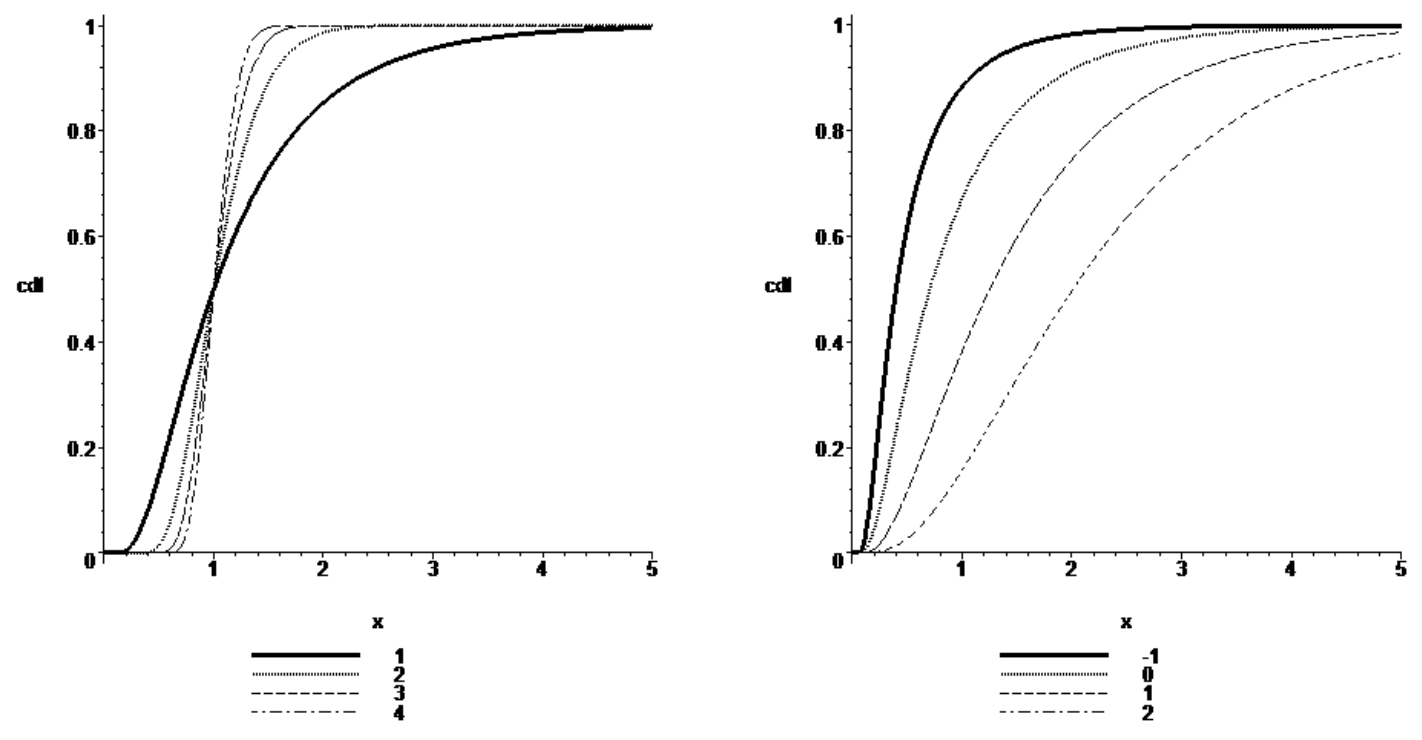

Figure 3: CDF plots of $X$ for $\alpha=1, \beta=1, \nu=0, p=1,2,3,4$ (left), and for $\alpha=1$, $\beta=1 / 2, p=1, \nu=-1,0,1,2$ (right).
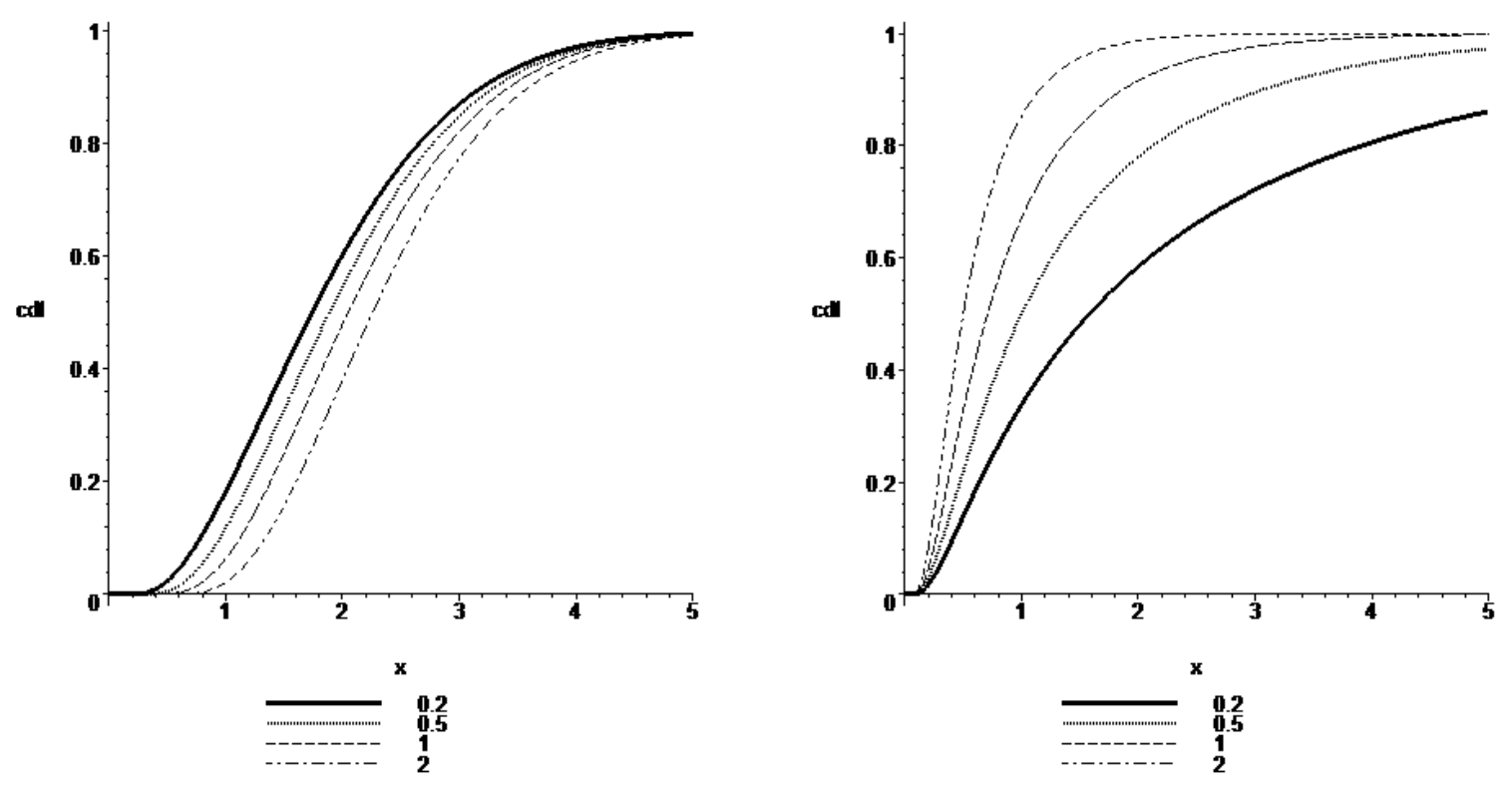

Figure 4: CDF plots of $X$ for $\alpha=0.2, \nu=3 / 2, p=2, \beta=0.2,0.5,1,2$ (left), and for $\beta=1 / 2, \nu=0, p=1, \alpha=0.2,0.5,1,2$ (right).

\section{Properties of the New Distribution}

\subsection{Mode}

The mode is the value of $x$ for which the pdf $f_{X}(x)$ is maximal. Now, differentiating (6), we have

$$
\frac{d f_{X}(x)}{d x}=\frac{-\alpha p x^{p}+\beta p x^{-p}+\nu-1}{x} f_{X}(x)
$$


which, when equated to 0 , gives the mode of the newly proposed pdf to be

$$
x_{m}=\sqrt[p]{\frac{\nu-1+\sqrt{(\nu-1)^{2}+4 \alpha \beta p^{2}}}{2 \alpha p}}, \quad \alpha>0, \beta>0, p>0,
$$

which is obtained by solving the quadratic equation $\alpha p\left(x^{p}\right)^{2}-(\nu-1) x^{p}-\beta p=0$, and ignoring the second root since, by our assumption, $x>0$. Differentiating (14), we get

$$
\frac{d^{2} f_{X}(x)}{d x^{2}}=\frac{1}{f_{X}(x)} \frac{d f_{X}(x)}{d x}\left[\frac{d f_{X}(x)}{d x}-x^{-1}\right]-p^{2} x^{-2}\left(\alpha x^{p}+\beta x^{-p}\right) .
$$

By simple arguments, it can easily be seen that

$$
\frac{d^{2}}{d x^{2}} f_{X}\left(x_{m}\right)<0
$$

Thus, the maximum value of the pdf (6) is given by $f_{X}\left(x_{m}\right)$. Clearly, the newly proposed pdf defined by (6) is unimodal.

\subsection{Moments}

\subsection{1 $\quad k$-th Moment (About the Origin)}

Suppose $X$ is a random variable with pdf $f_{X}(x)$ as given in (6). Then, using the Lemma 1 , the $k$-th moment, $\alpha_{k}$, of $X$, for some integer $k>0$, is easily expressed in terms of Macdonald function as

$$
\begin{aligned}
\alpha_{k} & =\mathrm{E}\left(X^{k}\right)=\int_{0}^{\infty} x^{k} f_{X}(x) d x \\
& =\left(\frac{\beta}{\alpha}\right)^{k / 2 p} \frac{K_{(k+\nu) / p}(2 \sqrt{\alpha \beta})}{K_{\nu / p}(2 \sqrt{\alpha \beta})}, \quad \alpha>0, \beta>0, k>0, p>0 .
\end{aligned}
$$

Using the definition of the Whittaker function, $\alpha_{k}$ is expressed as

$$
\alpha_{k}=2 \sqrt{\frac{\alpha}{\pi}}\left(\frac{\beta}{\alpha}\right)^{\frac{2 k+p}{4 p}} \frac{K_{(k+\nu) / p}(2 \sqrt{\alpha \beta})}{W_{0, \nu / p}(4 \sqrt{\alpha \beta})}, \quad \alpha>0, \beta>0, k>0, p>0 .
$$

Note: From equations (15) and (16), one can easily obtain the first, second and higher moments. The moments of different orders when $p=2$ and $\nu=1$ in (15) can be found in Chaudhry and Ahmad (1993).

\subsection{2 $k$-th Central Moment}

It is easy to see that the $k$-th central moment, $\beta_{k}$, of $X$ is

$$
\begin{aligned}
\beta_{k} & =\mathrm{E}(X-\mathrm{E}(X))^{k}=\int_{0}^{\infty}(x-\mathrm{E}(X))^{k} f_{X}(x) d x \\
& =\sum_{j=0}^{k}(-1)^{j}\left(\begin{array}{l}
k \\
j
\end{array}\right) \mathrm{E}^{j}(X) \mathrm{E}\left(X^{k-j}\right),
\end{aligned}
$$


where $\mathrm{E}^{j}(X)$ and $\mathrm{E}\left(X^{k-j}\right)$ are obtained by the equations (15) and (16). From (17), one can obtain the second, third, and higher central moments.

Note: Mean, Variance, Coefficients of Skewness and Kurtosis:

- Taking $k=1$ in (15) and (16), the mean $\alpha_{1}=\mathrm{E}(X)$ can be obtained.

- Taking $k=2$ in (17), the variance $\beta_{2}=\mathrm{E}(X-\mathrm{E}(X))^{2}$ is obtained.

- The Pearson's measure of skewness $\gamma_{1}$ and the kurtosis $\gamma_{2}$ are given by

$$
\gamma_{1}=\frac{\beta_{3}}{\beta_{2}^{3 / 2}} \quad \text { and } \quad \gamma_{2}=\frac{\beta_{4}}{\beta_{2}^{2}},
$$

where the variance $\beta_{2}$ and the third and fourth central moments $\beta_{3}$ and $\beta_{4}$ are obtained from (17) by taking $k=2, k=3$, and $k=4$, respectively.

Using a Maple 11 program, numerical values of the skewness $\gamma_{1}$ and the kurtosis $\gamma_{2}$ for some selected values of the parameters are provided in the Tables 1 to 3 . It is evident from these computations that the skewness is positive which implies that distribution of $X$ is positively skewed. Moreover, it is observed from these tables that for all selected values of the parameters the kurtosis $\gamma_{2}>3$ implying that the distribution is heavier tailed, except for the parameters $\alpha=1, \beta=1, \nu=2, p=4$, for which $\gamma_{2}=2.908<3$, implying that the distribution is lighter tailed.

Table 1: Moments for the parameters $\alpha=1, \beta=1, \nu=-1, p=1,2,3,4$

\begin{tabular}{cccccc}
\hline$p$ & $\beta_{2}$ & $\beta_{3}$ & $\beta_{4}$ & $\gamma_{1}$ & $\gamma_{2}$ \\
\hline 1 & 0.3369 & 0.4513 & 1.3785 & 2.3079 & 12.1449 \\
2 & 0.0982 & 0.0300 & 0.0415 & 0.9729 & 4.2984 \\
3 & 0.0450 & 0.0060 & 0.0068 & 0.6295 & 3.3795 \\
4 & 0.0256 & 0.0019 & 0.0020 & 0.4673 & 3.0864 \\
\hline
\end{tabular}

Table 2: Moments for the parameters $\alpha=1, \beta=1, \nu=0, p=1,2,3,4$

\begin{tabular}{cccccc}
\hline$p$ & $\beta_{2}$ & $\beta_{3}$ & $\beta_{4}$ & $\gamma_{1}$ & $\gamma_{2}$ \\
\hline 1 & 0.7200 & 1.1797 & 4.6966 & 1.9311 & 9.0607 \\
2 & 0.1191 & 0.0356 & 0.0560 & 0.8657 & 3.9504 \\
3 & 0.0490 & 0.0061 & 0.0078 & 0.5657 & 3.2471 \\
4 & 0.0268 & 0.0019 & 0.0022 & 0.4213 & 3.0158 \\
\hline
\end{tabular}

Table 3: Moments for the parameters $\alpha=1, \beta=1, \nu=2, p=1,2,3,4$

\begin{tabular}{cccccc}
\hline$p$ & $\beta_{2}$ & $\beta_{3}$ & $\beta_{4}$ & $\gamma_{1}$ & $\gamma_{2}$ \\
\hline 1 & 2.1450 & 4.1439 & 26.0664 & 1.3190 & 5.6652 \\
2 & 0.1598 & 0.0422 & 0.0880 & 0.6600 & 3.4471 \\
3 & 0.0560 & 0.0058 & 0.0095 & 0.4403 & 3.0473 \\
4 & 0.0289 & 0.0016 & 0.0024 & 0.3303 & 2.9080 \\
\hline
\end{tabular}




\subsection{Characteristic Function and $r$-th Cumulant}

It is easy to see that the characteristic function of $X$ is given by

$$
M_{X}(i t)=\mathrm{E}\left(e^{i t X}\right)=\sum_{k=0}^{\infty} \frac{(i t)^{k}}{k !} \mathrm{E}\left(X^{k}\right),
$$

where $i=\sqrt{-1}$ is the imaginary number $\left(i^{2}=-1\right)$ and $\mathrm{E}\left(X^{k}\right)$ denotes the $k$-th moment about the origin of $X$, which can be obtained from (15) and (16). The $r$-th cumulant $\kappa_{r}$ of $X$ having the characteristic function (18) is given by

$$
\kappa_{r}=\frac{1}{i^{r}}\left[\frac{d^{r}}{d t^{r}} \log f_{X}(t)\right]_{t=0}, \quad r=1,2, \ldots,
$$

from which, by successive differentiation, it can be seen that

$$
\kappa_{1}=\mathrm{E}(X)=\alpha_{1}, \quad \kappa_{2}=\operatorname{var}(X)=\beta_{2}, \quad \kappa_{3}=\mathrm{E}(X-\mathrm{E}(X)]^{3}=\beta_{3}, \text { etc. }
$$

\subsection{Entropy}

An entropy provides an excellent tool to quantify the amount of information (or uncertainty) contained in a random observation regarding its parent distribution (population). A large value of entropy implies the greater uncertainty in the data. As proposed by Shannon (1948), entropy of an absolutely continuous random variable $X$ having pdf $\phi_{X}(x)$ is defined as

$$
H(X)=\mathrm{E}\left(-\log \left(\phi_{X}(X)\right)=-\int_{S} \phi_{X}(x) \log \left(\phi_{X}(x)\right) d x\right.
$$

where $S=\left\{x: \phi_{X}(x)>0\right\}$. Thus, in view of (19), the entropy of $X$ having pdf (6) with normalizing constant $C$ given by (5) is expressed as

$$
\begin{aligned}
H(X) & =\mathrm{E}\left(-\log \left(f_{X}(X)\right)=-\int_{0}^{\infty} \log \left(f_{X}(x)\right) f_{X}(x) d x\right. \\
& =\alpha \mathrm{E}\left(X^{p}\right)+\beta \mathrm{E}\left(X^{-p}\right)-(\nu-1) \mathrm{E}(\log X)-\log (C) .
\end{aligned}
$$

Special Case: Suppose $X$ is a random variable with pdf $f_{X}(x)$ as given in (6), where $p=1, \nu=0, \alpha=\mu / a, \beta=\mu a,[a>0, \operatorname{Re}(\mu)>0]$. Then, applying the Equation (4.356.1), Page 577, in Gradshteyn and Ryzhik (2000), it can be seen that the expression for the entropy as given in (20) reduces to the following interesting form:

$$
H(X)=\log \left(2 a K_{0}(2 \mu)\right)+2 \mu \frac{K_{1}(2 \mu)}{K_{0}(2 \mu)}, \quad a>0, R e(\mu)>0 .
$$

\subsection{Survival and Hazard Functions}

The survival and hazard functions of the proposed distribution are respectively given by

$$
S(x)=1-F_{X}(x)=1-\frac{\gamma\left(\frac{\nu}{p}, \alpha x^{p} ; \alpha \beta\right)}{2(\alpha \beta)^{\frac{\nu}{2 p}} K_{\nu / p}(2 \sqrt{\alpha \beta})},
$$


and

$$
h(x)=\frac{f_{X}(x)}{1-F_{X}(x)}=\frac{\frac{p}{2}\left(\frac{\alpha}{\beta}\right)^{\frac{\nu}{2 p}} \frac{x^{\nu-1} \exp \left(-\alpha x^{p}-\beta x^{-p}\right)}{K_{\nu / p}(2 \sqrt{\alpha \beta})}}{1-\frac{\gamma\left(\frac{\nu}{p}, \alpha x^{p} ; \alpha \beta\right)}{2(\alpha \beta)^{\frac{\nu}{2 p}} K_{\nu / p}(2 \sqrt{\alpha \beta})}}
$$

where $x>0, \alpha>0, \beta>0, \nu \in \mathbb{R}$, and $p>0$. The possible shapes of the hazard function (23) are provided in Figure 5 for some selected values of the parameters.

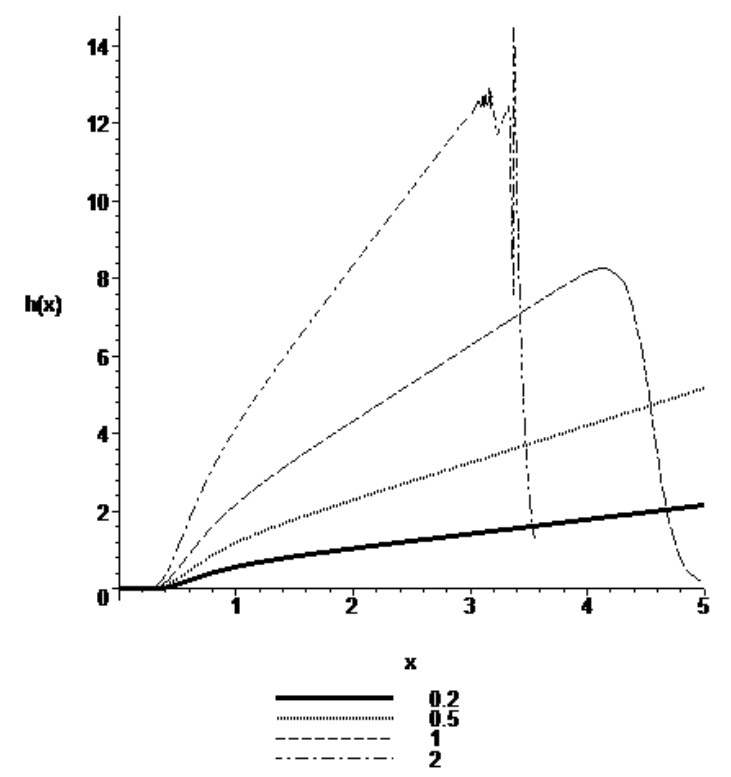

Figure 5: Plots of the hazard function $h(x)$ defined in (23) for $\beta=1 / 2, \nu=1, p=2$, and $\alpha=0.2,0.5,1,2$.

\subsection{Estimation of the Parameters}

\subsubsection{The Method of Maximum Likelihood}

Given a sample $\left\{x_{i}\right\}, i=1, \ldots, n$, the likelihood function corresponding to (6) is given by $L=\prod_{i=1}^{n} f\left(x_{i}\right)$. The objective of the likelihood function approach is to determine those values of the parameters that maximize $L$. Suppose $R=\log (L)=\sum_{i=1}^{n} \log \left(f\left(x_{i}\right)\right)$. Then the maximum likelihood estimates of the parameters $\alpha, \beta, \nu$, and $p$ are obtained by solving the maximum likelihood equations

$$
\frac{\partial R}{\partial \alpha}=0, \quad \frac{\partial R}{\partial \beta}=0, \quad \frac{\partial R}{\partial \nu}=0, \quad \text { and } \quad \frac{\partial R}{\partial p}=0
$$


From these, upon differentiation, the following systems of equations are obtained:

$$
\begin{aligned}
\frac{\partial R}{\partial \alpha} & =\sum_{i=1}^{n}\left[\frac{\nu}{2 \alpha p}-x_{i}^{p}-\sqrt{\frac{\beta}{\alpha}} \frac{K_{\nu / p}^{\prime}(2 \sqrt{\alpha \beta})}{K_{\nu / p}(2 \sqrt{\alpha \beta})}\right]=0 \\
\frac{\partial R}{\partial \beta} & =\sum_{i=1}^{n}\left[\frac{\nu}{2 \beta p}+x_{i}^{-p}+\sqrt{\frac{\alpha}{\beta}} \frac{K_{\nu / p}^{\prime}(2 \sqrt{\alpha \beta})}{K_{\nu / p}(2 \sqrt{\alpha \beta})}\right]=0 \\
\frac{\partial R}{\partial \nu} & =\sum_{i=1}^{n}\left[\frac{1}{2 p} \log \frac{\alpha}{\beta}+\log \left(x_{i}\right)-\frac{1}{p K_{\nu / p}(2 \sqrt{\alpha \beta})} \frac{\partial}{\partial(\nu / p)} K_{\nu / p}(2 \sqrt{\alpha \beta})\right]=0 \\
\frac{\partial R}{\partial p} & =\sum_{i=1}^{n}\left[\frac{1}{p}-\frac{\nu}{2 p^{2}} \log \frac{\alpha}{\beta}-\alpha p x_{i}^{p-1}+\beta p x_{i}^{-p-1}+\frac{\nu}{p^{2} K_{\nu / p}(2 \sqrt{\alpha \beta})} \frac{\partial K_{\nu / p}(2 \sqrt{\alpha \beta})}{\partial(\nu / p)}\right] \\
& =0
\end{aligned}
$$

where the derivatives $K_{\lambda}^{\prime}(\cdot)$ and $\partial K_{\lambda}(\cdot) / \partial \lambda$ of the modified Bessel function $K_{\lambda}(\cdot)$ of the third kind with respect to the argument and index (or order) $\lambda$, respectively, may be computed using the analytical formulas provided in Abramowitz and Stegun (1970) and Gradshteyn and Ryzhik (2000). Note that when $p=1$ and $\nu=-1 / 2$, the maximum likelihood estimates of the parameters $\alpha$ and $\beta$ can be found in Koutrouvelis, Canavos, and Meintanis (2005). For $p=1$ and $\nu \in \mathbb{R}$, equation (6) reduces to the pdf of the GIG distribution. For the maximum likelihood estimates of the parameters $\alpha, \beta$ and $\nu$ of the GIG distribution, the interested readers are referred to Jørgensen (1982). When $p=2$ and $\nu=1$, the maximum likelihood estimates of the parameters $\alpha$ and $\beta$ can be found in Chaudhry and Ahmad (1993). When $p>2$ and $\nu \in \mathbb{R}$, the maximum likelihood estimates of the parameters $\alpha, \beta, \nu$, and $p$ are determined by solving equations (24) to (27) following the iteration methods as developed in Lee (2010) and using Maple 11.

\subsubsection{The Method of Moments}

The first three moments of the random variable $X$ with pdf $f_{X}(x)$ in (6) are given by

$$
\alpha_{k}=\mathrm{E}\left(X^{k}\right)=\left(\frac{\beta}{\alpha}\right)^{\frac{k}{2 p}} \frac{K_{(k+\nu) / p}(2 \sqrt{\alpha \beta})}{K_{\nu / p}(2 \sqrt{\alpha \beta})},
$$

where $\nu \in \mathbb{R}, \alpha>0, \beta>0, p>0$, and $k=1,2,3,4$. Since the moment equation (28) depends on the modified Bessel function $K_{\lambda}(\cdot)$ of the third kind, the moment estimation of the parameters $\alpha, \beta, \nu$, and $p$ are determined by solving the system of equations (28) following the Newton-Raphson iteration method and using Maple 11.

\section{Distributional Relationships}

It is easy to see that, by a simple transformation of the variable $X$ or by taking special values of the parameters $\alpha, \beta, \nu, p$ in equation (6), a number of distributions as given below, including those of Chaudhry and Ahmad (1993) and Chou and Huang (2004), are special cases of our proposed distribution. Thus, our distribution defines a new family 
(or a generalization) of GIG distributions. The details of the derivations of the following distributional relationships can be directly obtained from the authors.

1. $p$-th root reciprocal IG distribution.

2. Root reciprocal IG distribution.

3. Good's GIG distribution, see for example Good (1953), Wise (1975), and Jørgensen (1982), among others.

4. For $\nu=1, p=2$ equation (6) reduces to the pdf of the GIG distribution of Chaudhry and Ahmad (1993).

5. $p$-th root of the GIG distribution: define $X=\sqrt[p]{Y}$, the $p$-th root of the GIG random variable $Y$. Then it can be seen that the pdf of $X$ is given by (6), which coincides with those of Silva, Lopes, and Migon (2006) by suitably changing the parameters.

6. Relationship to GIG distributions in Seshadri and Wesolowski (2001), Wesolowski (2002), and Chou and Huang (2004): taking $p=1$ and $\nu=-\omega, 0<\omega<\infty$, in (6), we obtain the GIG distributions of Wesolowski (2002), and Chou and Huang (2004), with pdf as follows:

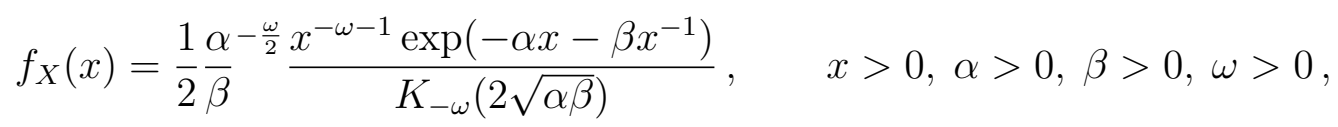

which reduces to the Seshadri and Wesolowski (2001) with $\alpha=2 a$ and $\beta=2 b$, where $a>0, b>0$.

7. Relationship to the mixing distribution in Gneiting's normal scale mixture: Gneiting (1997) defines a class of normal scale mixtures whose mixing distribution coincides with our newly proposed distribution by taking $p=1$ and suitably changing the parameters in (6). For more on normal scale mixtures and related literature, the interested readers are referred to the paper of Gneiting (1997).

8. Relationships to other distributions: It can be shown that by a simple transformation of the variable $x$ or by taking special values of the parameters $\alpha, \beta, \nu, p$, the following product pdf's are special cases (6):

(a) the product of the pdf's of half-normal and inverse half-normal distributions,

(b) the product of the pdf's of Rayleigh and inverse Rayleigh distributions,

(c) the product of the pdf's of Maxwell and inverse Maxwell distributions

(d) the product of the pdf's of chi and inverse chi distributions,

(e) the product of the pdf's of gamma and inverse gamma distributions,

(f) the product of the pdf's of Weibull and inverse Weibull distributions, and

(g) the product of the pdf's of root reciprocal exponential and Maxwell distributions, among others. 


\section{Percentiles}

This section computes the percentage points of the distribution with pdf given in (6). For any $0<q<1$, the $100 q$-th percentile (also called the quantile of order $q$ ) is a number $x_{q}$ such that the area under $f_{X}(x)$ to the left of $x_{q}$ is $q$. That is, $x_{q}$ is any root of the equation

$$
F\left(x_{q}\right)=\int_{-\infty}^{x_{q}} f_{X}(u) d u=q
$$

By numerically solving the equation for the cdf in (7), percentage points $x_{q}$ associated with the cdf of $X$ are computed for some selected values of the parameters. These are provided in the Tables 4 to 7 .

Table 4: Percentage points for $\alpha=1, \beta=1, \nu=0, p=1,2,3,4$

\begin{tabular}{ccccccc}
\hline$p$ & $75 \%$ & $80 \%$ & $85 \%$ & $90 \%$ & $95 \%$ & $99 \%$ \\
\hline 1 & 1.5677 & 1.7485 & 1.9822 & 2.3137 & 2.8874 & 4.2540 \\
2 & 1.2521 & 1.3223 & 1.4079 & 1.5211 & 1.6992 & 2.0625 \\
3 & 1.1617 & 1.2047 & 1.2562 & 1.3226 & 1.4240 & 1.6203 \\
4 & 1.1190 & 1.1499 & 1.1866 & 1.2333 & 1.3035 & 1.4362 \\
\hline
\end{tabular}

Table 5: Percentage points for $\alpha=1, \beta=1 / 2, \nu=-1,0,1,2, p=1$

\begin{tabular}{ccccccc}
\hline$\nu$ & $75 \%$ & $80 \%$ & $85 \%$ & $90 \%$ & $95 \%$ & $99 \%$ \\
\hline-1 & 0.6665 & 0.7590 & 0.8836 & 1.0693 & 1.4135 & 2.3272 \\
0 & 1.1945 & 1.3556 & 1.5669 & 1.8712 & 2.4073 & 3.7142 \\
1 & 2.0191 & 2.2568 & 2.5599 & 2.9824 & 3.6968 & 5.3359 \\
2 & 3.0396 & 3.3439 & 3.7249 & 4.2453 & 5.1033 & 7.0031 \\
\hline
\end{tabular}

Table 6: Percentage points for $\alpha=0.2, \beta=0.2,0.5,1,2, \nu=3 / 2, p=2$

\begin{tabular}{ccccccc}
\hline$\beta$ & $75 \%$ & $80 \%$ & $85 \%$ & $90 \%$ & $95 \%$ & $99 \%$ \\
\hline 0.2 & 2.4585 & 2.6541 & 2.8892 & 3.1946 & 3.6641 & 4.5866 \\
0.5 & 2.5858 & 2.7760 & 3.0051 & 3.3033 & 3.7630 & 4.6701 \\
1 & 2.7268 & 2.9120 & 3.1353 & 3.4265 & 3.8763 & 4.7672 \\
2 & 2.9173 & 3.0970 & 3.3138 & 3.5967 & 4.0345 & 4.9050 \\
\hline
\end{tabular}

Table 7: Percentage points for $\alpha=0.2,0.5,1,2, \beta=0.5, \nu=0, p=1$

\begin{tabular}{ccccccc}
\hline$\alpha$ & $75 \%$ & $80 \%$ & $85 \%$ & $90 \%$ & $95 \%$ & $99 \%$ \\
\hline 0.2 & 3.2941 & 3.9195 & 4.7707 & 6.0456 & 8.3920 & 14.4138 \\
0.5 & 1.8375 & 2.1258 & 2.5096 & 3.0716 & 4.0804 & 6.5955 \\
1 & 1.1945 & 1.3556 & 1.5669 & 1.8712 & 2.4073 & 3.7142 \\
2 & 0.7838 & 0.8742 & 0.9911 & 1.1568 & 1.4437 & 2.1270 \\
\hline
\end{tabular}

\section{Applications}

To illustrate the performance of our distribution, an example of tree circumferences in Marshall, Minnesota (based on data from Rice, 1999), has been considered in this section. 
The observed values are: $1.8,1.9,1.8,2.4,5.1,3.1,5.5,5.1,8.3,13.7,5.3,4.9,3.7,3.8$, 4.0, 3.4, 5.2, 4.1, 3.7, 3.9.

The mean, median and skewness of this data are 4.535, 3.950 and 2.118 respectively. We can see that the data is right skewed. Maple 11 has been used for computing the data moments, estimating the parameter (by employing the method of moments), and chi-square test for goodness-of-fit. The data moments are computed as $\hat{\mu}=4.5350$, $\hat{\mu}_{2}=27.2425$ and $\hat{\mu}_{3}=221.5905$. The estimation of the parameters and chi-square goodness-of-fit test are provided in Table 8 and 9, respectively.

Table 8: Parameter Estimates for the tree measurements data assuming different models

\begin{tabular}{lccc}
\hline $\operatorname{GGIG}(\nu, \alpha, \beta, p)$ & $\mathrm{IG}(\omega, \xi)$ & $\log -\operatorname{normal}(\mu, \sigma)$ & $\operatorname{Gamma}(\theta, r)$ \\
\hline$\hat{\nu}=\hat{p}=\hat{\alpha}=1$ & $\hat{\omega}=13.97$ & $\hat{\mu}=1.373$ & $\hat{\theta}=0.6793$ \\
$\hat{\beta}=14.16$ & $\hat{\xi}=4.535$ & $\hat{\sigma}=0.530$ & $\hat{r}=3.080$ \\
\hline
\end{tabular}

Table 9: Comparison criteria (chi-square test for goodness-of-fit)

\begin{tabular}{lcccc}
\hline & GGIG & IG & Log-normal & Gamma \\
\hline Test statistic & 2.766 & 6.378 & 5.822 & 7.208 \\
Critical value & 7.815 & 7.815 & 7.815 & 7.815 \\
p-value & 0.429 & 0.095 & 0.121 & 0.066 \\
\hline
\end{tabular}

From the chi-square goodness-of-fit test we observed that the GGIG, IG, log-normal, and gamma model fit the tree measurements data reasonably well. However, model GIG produces the highest p-value and therefore fits better than a IG, log-normal, and gamma distribution. Also, for the parameters estimated in Table 8, the GGIG, IG, log-normal, and gamma model have been superimposed on the histogram of the tree measurements data as in Figure 6 from which we observed that our GGIG model fits the tree measurements data reasonably well.

\section{Concluding Remarks}

This paper has derived a new family of distributions based on the generalized Pearson differential equation and named as GGIG distribution. Some characteristics of the newly proposed distribution are obtained. The infinite divisibility property of the newly proposed distribution family is discussed. The distributional relationships to some distributions are discussed. The plots for the cdf, pdf and hazard function, percentile points and tables for Pearson's measure of skewness and kurtosis for selected coefficients and parameters have been provided. The maximum likelihood and method of moment estimates of the parameters are discussed. It is observed that the new distribution is skewed to the right and bears most of the properties of skewed distributions. The statistical applications of the results to a problem of forestry from ecological science have been provided. It is found that the GGIG distribution fits better than gamma, log-normal and IG distributions. 


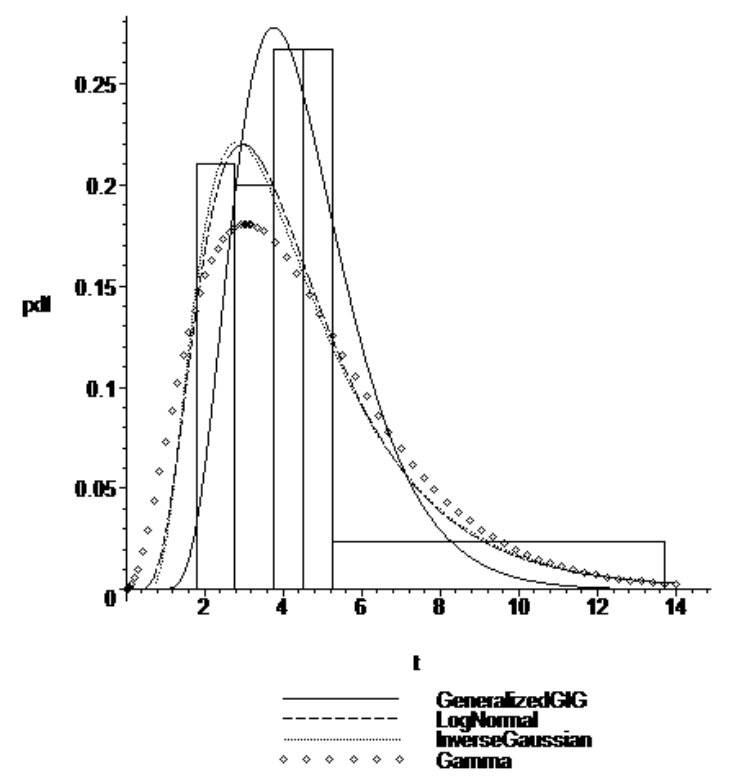

Figure 6: Fitting of the pdfs of the GGIG, IG, log-normal, and gamma model to the tree measurements data

\section{Acknowledgements}

The authors are thankful to the referees and the editor for their valuable comments and suggestions which greatly improved quality and presentation of this paper. We also acknowledge Prof. M. Ahsanullah, Prof. G. G. Hamedani, and Prof. H. Volkmer for providing useful hints about the infinite divisibility property of the above-mentioned distribution family.

\section{Appendix: Some Useful Mathematical Functions and Lemmas}

For details about these functions and lemmatas, see e.g. Abramowitz and Stegun (1970), Gradshteyn and Ryzhik (2000), Lebedev (1972), Prudnikov, Brychkov, and Marichev (1986), and Chaudhry and Zubair (2002), among others. The integrals

$\gamma(\alpha, x)=\int_{0}^{x} t^{\alpha-1} \exp (-t) d t, \quad$ and $\quad \Gamma(\alpha, x)=\int_{x}^{\infty} t^{\alpha-1} \exp (-t) d t, \quad \alpha>0$ are called incomplete gamma and complementary incomplete gamma function. Note that

$$
\frac{\partial \gamma(a, t)}{\partial t}=-\frac{\partial \Gamma(a, t)}{\partial t}=t^{a-1} \exp (-t)
$$

The integrals

$\gamma(\alpha, x ; b)=\int_{0}^{x} t^{\alpha-1} \exp \left(-t-b t^{-1}\right) d t \quad$ and $\quad \Gamma(\alpha, x ; b)=\int_{x}^{\infty} t^{\alpha-1} \exp \left(-t-b t^{-1}\right) d t$

are called the generalized incomplete gamma functions, where $\alpha, x$ are complex parameters and $b$ is a complex variable. When the argument $b=0$, the generalized incomplete 
gamma functions reduce to the ordinary incomplete gamma functions. The functions defined by

$$
\operatorname{erf}(x)=\frac{2}{\sqrt{\pi}} \int_{0}^{x} \exp \left(-u^{2}\right) d u \quad \text { and } \quad \operatorname{erfc}(x)=\frac{2}{\sqrt{\pi}} \int_{x}^{\infty} \exp \left(-u^{2}\right) d u=1-\operatorname{erf}(x)
$$

are called error and complementary error functions, respectively. The function defined by

$$
K_{\nu}(z)=\frac{1}{2}\left(\frac{z}{2}\right)^{\nu} \int_{0}^{\infty} t^{-\nu-1} \exp \left(-t-z^{2} / 4 t\right) d t, \quad|\arg z|<\pi / 2, \operatorname{Re}\left(z^{2}\right)>0
$$

is known as the modified Bessel function of the third kind or the Macdonald function) of index (or order) $\nu$. It is well known that

$$
K_{\nu}(z)=K_{-\nu}(z), \quad K_{ \pm 1 / 2}(z)=\sqrt{\frac{\pi}{2 z}} \exp (-z), \quad K_{\nu}(z)=\left(\frac{\pi}{2 z}\right)^{1 / 2} W_{0, \nu}(2 z)
$$

where $W_{\kappa, \nu}(\cdot)$ denotes the Whittaker function.

Lemma 1 Gradshteyn and Ryzhik (2000, equation (3.478.4), p. 342)

For $\operatorname{Re}(\alpha)>0$ and $\operatorname{Re}(\beta)>0$, we have

$$
\int_{0}^{\infty} x^{\nu-1} \exp \left(-\alpha x^{p}-\beta x^{-p}\right) d x=\frac{2}{p}\left(\frac{\beta}{\alpha}\right)^{\nu / 2 p} K_{\nu / p}(2 \sqrt{\alpha \beta}) .
$$

Lemma 2 Chaudhry and Zubair (2002, equation (2.120), p. 51).

$$
\Gamma(1 / 2, x ; b)=\frac{\sqrt{\pi}}{2}[\exp (-2 \sqrt{b}) \operatorname{erfc}(\sqrt{x}-\sqrt{b / x})+\exp (2 \sqrt{b}) \operatorname{erfc}(\sqrt{x}+\sqrt{b / x})] .
$$

\section{References}

Abramowitz, M., and Stegun, I. A. (1970). Handbook of Mathematical Functions, with Formulas, Graphs, and Mathematical Tables. New York: Dover.

Achcar, J. A., and Bolfarine, H. (1986). The log-linear model with generalized gamma distribution for the error: a Bayesian approach. Statistics and Probability Letters, $4,325-332$.

Barndorff-Nielson, O. E., and Halgreen, C. (1977). Infinite divisibility of the hyperbolic and generalized inverse Gaussian distributions. Zeitschrift für Wahrscheinlichkeitstheorie und verwandte Gebiete, 38, 309-311.

Chaudhry, M. A., and Ahmad, M. (1993). On a probability function useful in size modeling. Canadian Journal of Forest Research, 23, 1679-1683.

Chaudhry, M. A., and Zubair, S. M. (2002). On a Class of Incomplete Gamma Functions with Applications. Boca Raton: Chapman \& Hall/CRC.

Chou, C., and Huang, W. (2004). On characterizations of the gamma and generalized inverse gaussian distributions. Statistics and Probability Letters, 69, 381-388.

Dadpay, A., Soofi, E. S., and Soyer, R. (2007). Information measures for generalized gamma family. Journal of Econometrics, 138, 568-585. 
Dunning, K., and Hanson, J. N. (1977). Generalized Pearson distributions and nonlinear programming. Journal of Statistical Computation and Simulation, 6, 115-128.

Elderton, W. P. (1953). Frequency Curves and Correlation. Washington, D.C.: Harren Press.

Erdélyi, A., Magnus, W., Oberhettinger, F., and Tricomi, F. G. (1953). Higher Transcendental Functions, I. New York: McGraw-Hill.

Gneiting, T. (1997). Normal scale mixtures and dual probability densities. Journal of Statistical Computation and Simulation, 59, 115-128.

Good, I. J. (1953). The population frequencies of species and the estimation of population parameters. Biometrika, 40, 237-260.

Gradshteyn, I. S., and Ryzhik, I. M. (2000). Table of Integrals, Series, and Products (6th ed.). San Diego: Academic Press.

Johnson, N. L., Kotz, S., and Balakrishnan, N. (1994). Continuous Univariate Distributions (6th ed., Vol. 1). New York: John Wiley \& Sons.

Jørgensen, B. (1982). Statistical Properties of the Generalized Inverse Gaussian Distribution. New York: Springer Verlag.

Koutrouvelis, I. A., Canavos, G. C., and Meintanis, S. G. (2005). Estimation in the threeparameter inverse Gaussian distribution. Computational Statistics \& Data Analysis, 49, 1132-1147.

Lawless, J. F. (2003). Statistical Models and Methods for Lifetime Data (2nd ed.). New York: John Wiley \& Sons.

Lebedev, N. N. (1972). Special Functions and their Applications. New York: Dover.

Lee, J. (2010). Parameter estimation of the extended generalized Gaussian family distributions using maximum likelihood scheme. Information Technology Journal, 9 , 61-66.

Marshall, A. W., and Olkin, I. (2007). Life Distributions: Structure of Nonparametric, Semiparametric and Parametric Families. New York: Springer.

Prause, K. (1997). Modelling Financial Data Using Generalized Hyperbolic Distributions (Research Report No. 48). Universität Freiburg im Breisgau, Freiburg Center for Data Analysis and Modelling und Institut für Mathematische Stochastik.

Prause, K. (1999). The Generalized Hyperbolic Model: Estimation, Financial Derivatives, and Risk Measures. Unpublished doctoral dissertation, Universität Freiburg.

Prudnikov, A. P., Brychkov, Y. A., and Marichev, O. I. (1986). Integrals and Series (Vols. 1, 2, and 3). Amsterdam: Gordon and Breach Science Publishers.

Rice, S. (1999). Tree measurements: An outdoor activity for learning the principles of scaling. American Biology Teacher, 61, 677-679.

Seshadri, V., and Wesolowski, J. (2001). Mutual characterizations of the gamma and generalized inverse Gaussian laws by constancy of regression. Sankhya, A, 63, 107-112.

Shakil, M., Singh, J. N., and Kibria, B. M. G. (2010). On a family of product distributions based on the Whittaker functions and generalized Pearson differential equation. Pakistan Journal of Statistics, 26, 111-125.

Shannon, C. E. (1948). A mathematical theory of communication. Bell System Technical Journal, 27, 379-423, 623-656.

Silva, R. S., Lopes, H. F., and Migon, H. S. (2006). The extended generalized inverse 
Gaussian distribution for log-linear and stochastic volatility models. Brazilian Journal of Probability and Statistics, 20, 67-91.

Steutel, F. W., and Harn, V. K. (2004). Infinite Divisibility of Probability Distributions on the Real Line. New York: Marcel Dekker, Inc.

Stuart, A., and Ord, J. K. (1994). Kendall's Advanced Theory of Statistics (6th ed.). London: Edward Arnold.

Wesolowski, J. (2002). The Matsumoto-Yor independence property for GIG and gamma laws revisited. Mathematical Proceedings of Cambridge Philosophical Society, $133,153-161$.

Wise, M. G. (1975). Skew distributions in biomedicine including some with negative powers of time. In Model Building and Model Selection, Statistical Distributions in Scientific Work.

Authors' addresses:

Mohammad Shakil

Department of Mathematics

Miami Dade College, Hialeah Campus (Miami)

1800 West 49th Street, Suite 4311-4 Hialeah

Fl 33012, USA

E-mail: mshakil@mdc.edu

B. M. Golam Kibria

Department of Mathematics and Statistics

Florida International University

University Park

Miami, FL 33199

USA

E-mail: kibriag@fiu.edu

Jai Narain Singh

Department of Mathematics and Computer Science

Barry University

Miami Shores, FL 33161

USA

E-mail: jsingh@mail barry.edu 